\title{
Étude de la migration du soluté d'un lixiviat dans un sol non saturé par la méthode TDR
}

\section{ALIMI-ICHOLA} L. GAIDI

Laboratoire URGC-

Géotechnique

INSA de Lyon

Bât. J.C.A. Coulomb

$3^{e}$ étage 34, av. des Arts

69621 Villeurbanne

ibrahim.alimi-ichola@

insa-lyon.fr

laouni.gaidi@insa-lyon.fr
La protection de l'environnement nécessite la réalisation de systèmes étanches constitués de sol compacté pour le confinement des déchets. Afin de connaître les modes de migration de l'eau et des polluants dans ces systèmes, on présente les résultats d'une série d'essais d'infiltration dans une colonne de sol non saturé, équipée de sondes TDR.

La méthode TDR permet de suivre en même temps la teneur en eau et la conductivité électrique (ou l'impédance) d'un sol d'une manière continue. Des essais d'étalonnage des sondes sur six sols et trois lixiviats ont permis d'avoir de nouvelles relations entre la teneur en eau volumique $(\theta)$ du sol et sa constante diélectrique $(\varepsilon)$. Ces relations, plus précises que celle de Topp et al. (1980), sont utilisées pour déterminer les profils hydriques. La relation établie entre la conductivité électrique et la concentration en éléments solubles du lixiviat permet la détermination des profils de concentration et la courbe de sortie de ces éléments. Ces courbes sont utilisées pour le calcul de la vitesse de convection et du coefficient de dispersion apparent. Les résultats des essais d'infiltration montrent que la perméabilité du sol et la diffusivité sont plus élevées lors de l'infiltration du lixiviat. La vitesse d'infiltration et la vitesse de convection, au sens de Darcy, sont supérieures à la vitesse de sortie du lixiviat. Les trois vitesses tendent vers une valeur commune qui dépend de la perméabilité du sol. L'application du modèle de Green et Ampt (1911) à l'infiltration montre que le front d'humidité avance plus vite que le front de pollution.

La méthode statistique (Mermoud, 1980) est appliquée aux profils d'humidité et aux profils de concentration pour le calcul du coefficient de dispersion apparent et de la vitesse de convection du soluté au cours de la saturation du sol. On remarque que le coefficient apparent de dispersion augmente au cours de la saturation. L'utilisation de la courbe de conductivité électrique du percolat et de la courbe d'évolution de la fraction soluble donne les mêmes résultats de calcul du coefficient de dispersion apparent.

L'utilisation des mesures TDR a permis la comparaison des paramètres de migration de l'eau et de soluté. On peut envisager le développement d'une méthode de mesure in situ de la perméabilité par infiltration.

Mots-clés : méthode TDR, sol non saturé, teneur en eau, succion, constante diélectrique, sorption. 


\section{Study of the solute migration through unsaturated soil using the TDR method}

The protection of the environment requires building waste containment systems made up of liners of compacted soil. In this paper, infiltration tests are carried out on soil columns equipped with TDR probes to investigate how water and pollutants migrate in the liners.

The TDR method is a technique to measure simuitaneously the volumetric water content and the electrical conductivity (or the impedance) of the soil in a continuous manner. Using six soils and three different leachates, new relationships between the volumetric water content $(\theta)$ of the soil and its dielectric constant $(\varepsilon)$ is established. These relationships appear to be more accurate than the one of Topp ef al. (1980). They are used to determine water content profles in a soil column. The relationship between the leachate solute concentration and the electrical conductivity allows to compute the solute concentration profiles in the soll column and the solute breakthrough curve. These curves are used to determine solute convection rate and solute apparent dispersion coefficient. Infiitration test results show that the soil permeability and the diffusivity increase when the leachate is infiltrated. The infiltration rate and the solute convection rate are higher than the leachate outflow rate and the three rates yield to a same value which depends on the soll permeability. The Green and Ampt (1911) model applied to the infiltration shows that the movement of the wet front is faster than the movement of the pollution front.

The apparent dispersion coefficient and the solute convection rate are computed by applying the statistic distribution law to the moisture and the solute concentration profiles. It is observed that the apparent dispersion coefficient increases during the saturation of the sample. The electrical conductivity and the solute concentration of the percolated leachate are scaled with the initial values. The evolution curves of these scaled values with elapsed time are used to compute the apparent dispersion coefficient. There is little difference between the apparent dispersion values obtained with the scaled electrical conductivity evolution curve and the scaled solute concentration evolution curve

The use of the TDR measurements allows comparison between solute and water migration parameters and the obtained results can be applied to in situ permeablity measurements.

Key words: TDR method, unsaturated soil, water content, suction, dielectric constant, sorption.

\section{Introduction}

La protection de l'environnement exige la construction de centres de stockage et de bassins de traitement des effluents. Les systèmes d'étanchéité de ces ouvrages ont fait l'objet d'importantes recherches sur leur capacité à limiter le flux de polluants. Mais la qualification des matériaux utilisés se fait uniquement par leur coefficient de perméabilité et ne prend pas en compte toute les propriétés liées à l'état de saturation des matériaux qui participent à la limitation de ce flux.

L'étude de la migration des polluants contenus dans un liquide dans un sol non saturé a pour but la description des modes de migration et la détermination des paramètres qui régissent le transfert. Ce transfert étant lié à celui de l'eau, il faut se référer aux études des écoulements dans les sols non saturés, qui ont permis la mise au point des méthodes de mesure des paramètres caractéristiques. Des modèles de conductivités hydrauliques et de profils hydriques permettent la prévision du mouvement d'eau et de la position de l'humidité dans la zone non saturée au cours d'une infiltration. Des chercheurs (Laryea et al, 1982; Elrick et al., 1983) ont alors appliqué les résultats de l'écoulement de l'eau pour décrire celui des polluants dans les milieux poreux non saturé. Pour comprendre le mouvement des polluants résidant ou arrivant dans une zone non saturée et le différencier de celui de l'eau, il est nécessaire de disposer d'une technique de suivi du polluant et de l'humidité dans cette zone.

Dans la zone non saturée, la migration des polluants est liée à la variation de la teneur en eau. Il existe une abondante littérature concernant la mesure de la teneur en eau. Plusieurs chercheurs dont (Topp et al., 1980) ont montré au début des années 80 qu'il était possible de mesurer la teneur en eau volumique d'un 
sol $\theta$ (volume d'eau/volume du sol), en utilisant la réflectométrie dans le domaine temporel (TDR: time domain reflectometry). La conductivité électrique du sol dépend de la charge en ions de l'eau interstitielle. Cette charge en ions provoque l'atténuation du signal TDR et la mesure de cette atténuation sert à déterminer la conductivité électrique du sol. La rapidité des mesures par cette technique permet de suivre en temps réel la variation de l'humidité et de la conductivité électrique en un point choisi.

Dans ce travail, des essais d'infiltration d'un lixiviat de scorie de plomb sont réalisés sur des colonnes de sol équipées de sondes TDR pour obtenir les profils hydriques et les profils d'impédance. On déduit des profils d'impédance les profils de conductivité électrique. Au cours de l'infiltration, une mesure des volumes du liquide entrant et sortant est réalisée au cours du temps ainsi que celle de la conductivité électrique du percolat. Le débit de liquide sortant de la colonne donne le coefficient de perméabilité du sol. Des essais de sorption effectués sur les échantillons du sol sont utilisés pour établir la loi d'adsorption et déterminer le coefficient de distribution $\mathrm{k}_{\mathrm{d}}$ de l'ensemble des éléments solubles. Les résultats de tous ces essais servent à décrire le mouvement de l'eau et du soluté dans la colonne de sol.

\section{2}

\section{Transferts d'eau dans un milieu poreux non saturé}

\section{Transferts d'eau dans un milieu poreux non saturé}

L'écoulement de l'eau dans les sols saturés est décrit par la lof de Darcy. Cette loi a été modifiée par plusieurs chercheurs pour son application aux mouvements de l'eau dans les sols non saturés (Buckingham. 1907 ; Richards, 1931: Childs et Collis-George, 1950). Elle est alors écrite sous la forme généralisée:

$$
q=-K(\theta) \text { grad } h
$$

où:

$q$ est le flux, débit d'eau par unité de surface, qui traverse la zone non saturée $\left(\mathrm{m}^{3} / \mathrm{m}^{2} / \mathrm{s}\right)$;

$K(\theta)$ est la conductivité hydraulique du milieu poreux non saturé $(\mathrm{m} / \mathrm{s})$, fonction de la teneur en eau volumique $\theta$;

h est le potentiel de l'eau dans le sol exprimé en mètre d'eau (m).

En absence d'effet thermique et d'effet osmotique, le potentiel h de l'eau dans le sol est la somme de deux termes

- le potentiel matriciel ou succion matricielle s exprimée en $\mathrm{kPa}$;

- le potentiel gravitaire $z(m)$ qui représente l'action de la pesanteur;

soit:

$$
h=-\frac{u_{u}-u_{w}}{\gamma_{w}}+z=-\frac{S}{\gamma_{w}}+z
$$

où:

$u_{2}$ la pression d'air et $u_{w}$ la pression effective de l'eau.
La courbe de rétention est définie par la relation entre la succion $s$ et la teneur en eau volumique $\theta$. Childs et Collis-George (1948) ont défini la diffusivité de l'eau par:

$$
D(\theta)=-K(\theta) \frac{1}{\gamma_{w}} \frac{\partial s}{\partial \theta}
$$

Cette diffusivité est différente de la diffusion moléculaire de l'eau dans les pores. Elle permet la simplification du traitement mathématique des écoulements horizontaux dans les sols non saturés.

En introduisant dans l'équation de conservation de la masse la loi de Darcy généralisée (1), on obtient l'équation de Richards (1931) qui régit le mouvement d'eau dans les milieux poreux non saturés:

$$
\frac{\partial \theta}{\partial t}=-\frac{1}{\gamma_{u}} \operatorname{div}[K(\theta) \operatorname{grad}(s(\theta))]+\frac{\partial K}{\partial z}=\operatorname{div}[D(\theta) \operatorname{grad} \theta]+\frac{\partial K(\theta)}{\partial z}
$$

Dans le cas d'un écoulement horizontal unidirectionnel suivant la direction $x$ et en utilisant l'expression (3) de la diffusivité, l'équation (4) devient:

$$
\frac{\partial \theta}{\partial t}=D(\theta) \frac{\partial^{2} \theta}{\partial x^{2}}
$$

En associant à l'équation (5) les conditions aux limites suivantes:

- pour $t=0$ et $x>0, \theta=\theta$ la teneur en eau volumique initiale:

- pour $t \geq 0$ et $x=0, \theta=\theta_{0}$ la teneur en eau volumique finale;

et utilisant la transformation de Boltzmann: $x(\theta, t)=$ $\eta(\theta) \sqrt{t}$ où $\eta(\theta)$ est le profil hydrique, Philip (1955) a proposé une solution de l'équation (5) sous la forme:

$$
I=\int_{\Omega}^{n} x(\theta, t) d \theta=\int_{1}^{n} \eta(\theta) d \theta \sqrt{t}=S\left(\theta_{,}, \theta_{0}\right) \sqrt{t}
$$

$I(\mathrm{~m})$ est l'infiltration cumulée à l'instant $t$ dans le milieu poreux, lorsque la teneur en eau passe de $\theta$ à $\theta$. $S\left(\theta_{1}, \theta_{0}\right)$ est la sorptivité du milieu poreux. Elle dépend de $\theta$ et $\theta_{0}$ ou des succions correspondantes $\mathrm{s}_{\mathrm{i}}$ et $\mathrm{s}_{0}$. Philip utilise un développement du profil hydrique $z(\theta, t)$ en série de puissance de $\sqrt{t}$ pour obtenir la solution de l'équation (4) dans le cas d'un écoulement vertical. Pour assurer la convergence de cette série, Philip introduit un rayon de convergence estimé par le temps $t_{\text {prav }}$

$$
t_{\text {grav }}=\left(\frac{S\left(\theta_{1}, \theta_{0}\right)}{K_{0}-K_{1}}\right)^{2}
$$

อบ่:

$S\left(\theta_{p} \theta_{0}\right)$ est la sorptivité, $K_{0}$ est la conductivité hydraulique à $\theta_{0}$ et $\mathrm{K}$ la conductivité hydraulique à $\theta$;

$t_{\text {crav }}$ correspond au temps pour lequel l'influence des forces gravitaires et des forces capillaires sur le processus d'infiltration est du même ordre. Au-delà de $t_{\text {mav }}$ Philip propose une solution asymptotique de la forme:

$$
z(\theta, t)=z_{-}(\theta)+u_{f}(t)\left(t-t_{\text {(answ }}\right)
$$

où $z(\theta)$ correspond au profil hydrique pour $t$ très grand et $u_{f}(t)$ est la vitesse de translation de $z_{-}(\theta)$.

En utilisant la transformation de Boltzmann: $z(\theta, t)=$ $\eta(\theta) \sqrt{t}$, Green et Ampt (1911) définissent le profil hydrique par:

$$
\theta=\left\{\begin{array}{c}
\theta_{0}, \ldots .0 \leq \eta \leq \eta_{G} \\
\theta_{1}, \ldots . \eta \geq \eta_{G}
\end{array}\right.
$$

avec $\eta_{G}=\frac{S\left(\theta_{j}, \theta_{0}\right)}{\theta_{0}-\theta_{j}}$ valeur moyenne de $\eta$ entre $\theta$ et $\theta_{0}$. 
Pour une infiltration cumulée $\mathrm{I}_{z^{\prime}}$ la profondeur $\mathrm{z}_{\mathrm{C}}$ du front d'humidité est alors donnée par (Elrick et al., 1983) ;

$$
z_{G}=\frac{I_{2}}{\left(\theta_{0}-\theta_{i}\right)}
$$

L'équation (10) permet le suivi de la pénétration du front de l'humidité au cours de l'infiltration.

\section{2}

\section{Transfert de soluté dans un milieu poreux non saturé}

Le transfert de soluté dans un milieu poreux est décrit par un mouvement de convection et de dispersion. Le débit de soluté par unité de surface q, lorsque la concentration du soluté transporté est C, est donné par:

$$
q_{s}=-D \text { g gradC }+q C
$$

où $\mathrm{D}_{d}$ est le coefficient de dispersion du soluté, $\theta$ la teneur en eau volumique du milieu poreux, q le débit de liquide par unité de surface donné par l'équation (1) et $\mathrm{C}$ sa concentration en soluté.

Lorsqu'on admet qu'au cours du transfert de soluté il n'y a ni disparition ni apparition de matière, par application de la loi de conservation de la masse de soluté, l'équation de transfert de soluté dans le cas d'un mouvement unidirectionnel s'écrit :

$$
\frac{\partial(\theta C)}{\partial t}=\frac{\partial}{\partial x}\left(D_{d t} \theta \frac{\partial C}{\partial x}\right)-\frac{\partial}{\partial x}(q C)
$$

Lorsque le flux q et la teneur en volumique $\theta$ prennent des valeurs constantes, l'équation (12) devient:

$$
\frac{\partial C}{\partial t}=D_{d} \frac{\partial^{2} C}{\partial x^{2}}-u \frac{\partial C}{\partial x}
$$

où $u=\frac{q}{\theta}$ est appelé vitesse moyenne de pore qui représente la moyenne globale des vitesses microscopiques de l'eau dans le volume total du sol. Dans cette définition, on suppose que toute l'eau du sol participe au mouvement du soluté et correspond au flux de Darcy (Gaudet, 1978; Mermoud, 1982).

Dans certains sols, le bilan de masse est perturbé par les échanges entre la solution et la matrice poreuse. Cette dernière peut soit fixer les ions (adsorption), soit en libérer (désorption). Ce phénomène d'adsorptiondésorption est caractérisé par la cinétique et l'isotherme de sorption. Si S représente la concentration de la phase adsorbée par unité de masse de sol, en régime hydrodynamique permanent, l'équation (13) est alors remplacée par:

$$
\frac{\rho}{\theta} \frac{\partial S}{\partial t}+\frac{\partial C}{\partial t}=D_{d} \frac{\partial^{2} C}{\partial x^{2}}-u \frac{\partial C}{\partial x}
$$

avec $\rho$ : masse volumique apparente du sol.

La détermination de la fonction $\mathrm{S}$ dépend de la cinétique et des isothermes d'échanges. Les isothermes les plus utilisées sont les relations de Freundlich:

$$
\begin{gathered}
S=k_{d} C^{N} \\
\text { et l'isotherme linéaire (cas } N=1) \text { : } \\
S=k_{d} C
\end{gathered}
$$

$k_{\text {et }} \mathrm{N}$ sont des coefficients empiriques fonction du sol et du type de soluté.
En introduisant la relation (15) dans l'équation (14), l'équation de transfert d'un soluté avec sorption d'ions s'écrit:

$$
R(C) \frac{\partial C}{\partial t}=D_{d} \frac{\partial^{2} C}{\partial x^{2}}+u \frac{\partial C}{\partial x}
$$

en posant $R(C)=\frac{\rho}{\theta} N k_{d} C^{N-1}+1 \cdot R(C)$ est appelé la fonction de retard.

En associant à l'équation (14) les conditions aux limites suivantes:

- pour $t=0$ et $x>0, C=0$ la concentration initiale de soluté nulle dans l'eau interstitielle;

- pour $t \geq 0$ et $x=0, C=C_{0}$ la concentration du soluté dans la solution infiltrée;

la solution de l'équation de transfert de soluté par convection-dispersion s'écrit:

$$
\frac{C(x, t)}{C_{0}}=\frac{1}{2}\left[\operatorname{erfc}\left(\frac{x-u t}{\sqrt{4 D_{d} t}}\right)+\exp \left(\frac{u x}{D_{d t}}\right) \operatorname{erfc}\left(\frac{x+u t}{\sqrt{4 D_{d} t}}\right)\right]
$$

où erfc(x) est la fonction erreur complémentaire définie par:

$$
\operatorname{erfc}(x)=1-\operatorname{erf}(x)=\frac{2}{\sqrt{\pi}} \int_{x}^{-} \exp \left(-y^{2}\right) d y
$$

La relation (18) donne la distribution de la concentration du soluté dans l'eau interstitielle à l'instant $t$. Cette relation est utilisée pour la recherche des deux paramètres u (vitesse de pore) et $\mathrm{D}_{\mathrm{f}}$ (coefficient de dispersion) par calage aux mesures des profils de concentration. Le deuxième terme du second membre de la relation [18] étant petit devant le premier terme peut être négligé. La concentration relative $\underline{C(x, t)}$ est égale à:

$$
\frac{C(x, t)}{C_{0}}=\frac{1}{2} \operatorname{erfc}\left(\frac{x-u t}{\sqrt{4 D_{j} t}}\right)=\frac{1}{\sqrt{\pi}} \int_{\frac{x \rightarrow u}{\sqrt{2 D_{d} t}}}^{-\pi} e^{-\frac{1}{2} t s^{t} t} d s=1-F(z)
$$

avec: $F(x)=\frac{1}{\sqrt{2 \pi}} \int^{x} e^{-\frac{1}{2}\left(s^{2}\right)} d s$

La fonction $F$ définit une loi de Gauss sous la forme réduite $\left(S=\frac{x-\bar{x}}{\sigma}\right)$. Le rapport $\frac{C}{C_{0}}$ suit une loi de distribution $1-F(x)$ ayant pour valeur moyenne $\bar{x}=u t$ et pour écart type $\sigma=\sqrt{2 D_{d} t}$. La méthode statistique (Mermoud, 1978) utilise ces deux paramètres de distribution pour la détermination de la vitesse moyenne de pore $u$ et du coefficient de dispersion $D_{\text {c. }}$

Lorsque le transfert de soluté est purement convectif, la profondeur du soluté après une infiltration cumulée I est donnée par la relation (10). Lorsqu'il y a adsorption de soluté par la matrice poreuse au cours du transfert, Laryea et al. (1982) proposent, pour l'obtention de la profondeur du soluté, la relation suivante:

$$
z_{s}^{R}=\frac{I_{z}}{R \theta_{s}}
$$

$R$ est la fonction de retard et $\theta_{\mathrm{s}}$ la teneur volumique de saturation.

Le résumé bibliographique ci-dessus donne les éléments qui permettent la détermination des paramètres de transport de l'eau et du soluté dans un milieu poreux. L'utilisation de ces méthodes nécessite la connaissance des profils d'humidité et de concentration des solutés obtenus dans ce travail par la méthode TDR. 


\section{Matériels et méthodes}

\section{1}

\section{Caractérisation du sol d'essai}

Pour limiter la durée des essais, un sol dont la perméabilité n'est pas trop faible $\left(\mathrm{k}>10^{-8} \mathrm{~m} / \mathrm{s}\right)$ est recherché. Il doit présenter quelques propriétés d'adsorption pour observer l'influence des échanges avec la matrice poreuse sur le mouvement du soluté. Après des essais d'identification sur plusieurs sols et plusieurs échantillons, le tout-venant fourni par la CASEMA (BourgAchard) a été retenu. Les caractéristiques géotechniques du sol utilisé sont données dans les tableaux I et II. Les résultats des essais de compactage donnent une teneur en eau optimale de $12 \%$ pour une densité sèche de 1,9. D'après sa courbe granulométrie et ses limites d'Atterberg, le sol s'identifie à un sable argileux dans la classification LCPC.

Les échantillons utilisés sont écrêtés à $5 \mathrm{~mm}$. Pour obtenir une perméabilité de l'ordre $10^{-7} \mathrm{~m} / \mathrm{s}$, le sol d'essai est compacté à une densité sèche égale à 1,6.

\begin{tabular}{|c|c|c|c|}
\hline TABLEAUI & $\begin{array}{l}\text { Granulomé } \\
\text { Grain size dis }\end{array}$ & $\begin{array}{l}\text { du sol. } \\
\text { bution. }\end{array}$ & \\
\hline $\begin{array}{l}\text { Gravier } \\
\text { ( } 5 \mathrm{~mm}\}\end{array}$ & $\begin{array}{c}\text { Sable } \\
(>2 \mathrm{~mm})\end{array}$ & $\begin{array}{c}\text { Fines } \\
(<0,080 \mathrm{~mm})\end{array}$ & $\begin{array}{l}\text { Argile } \\
\text { (<2 } 2 \text { mm) }\end{array}$ \\
\hline $6 \%$ & $8 \%$ & $76 \%$ & $21 \%$ \\
\hline
\end{tabular}

TABLEAUII Paramètres de consistance (les limites d'Atterberg).

Soil consistance parameters (Atterberg limits).

\begin{tabular}{ccccc}
$\begin{array}{c}\text { Teneur } \\
\text { en eau } \\
\text { naturelle }\end{array}$ & $\begin{array}{c}\text { Limite de } \\
\text { liquidité } \\
W_{b}\end{array}$ & $\begin{array}{c}\text { Limite de } \\
\text { plasticité } \\
w_{L}\end{array}$ & $\begin{array}{c}\text { Indice de } \\
\text { plasticité } \\
I_{p}\end{array}$ & $\begin{array}{c}\text { Indice de } \\
\text { consistance } \\
I_{e}\end{array}$ \\
\hline $12 \%$ & $33 \%$ & $20 \%$ & $13 \%$ & $11 \%$
\end{tabular}

La succion du sol est mesurée à différentes teneurs en eau par la méthode du papier filtre et la méthode d'adsorption-désorption de la vapeur d'eau, pour la détermination de la courbe caractéristique de rétention. d'eau. L'ensemble des résultats de mesures et la courbe ajustée selon le modèle de Van Genuchten (1980) sont représentés sur la figure 1. L'équation semi-empirique qui donne la relation entre la teneur en eau volumique $\theta$ et la pression effective $h$ de l'eau du sol exprimée en centimètre d'eau est:

$$
\theta(h)=\theta_{r}+\frac{\theta_{s}-\theta_{r}}{\left(1+(\alpha h)^{n}\right)^{m}}
$$

$\theta_{r}$ : teneur en eau volumique résiduelle;

$\theta_{s}$ : teneur en eau volumique de saturation $(h=0)$; $\alpha, n$ et $m$ : les paramètres du modèle.

Avec ce modèle on détermine la teneur en eau volumique à $h$ nul $\left(\theta_{s}\right)$ et la teneur en eau résiduelle à forte succion $\theta_{r}$.

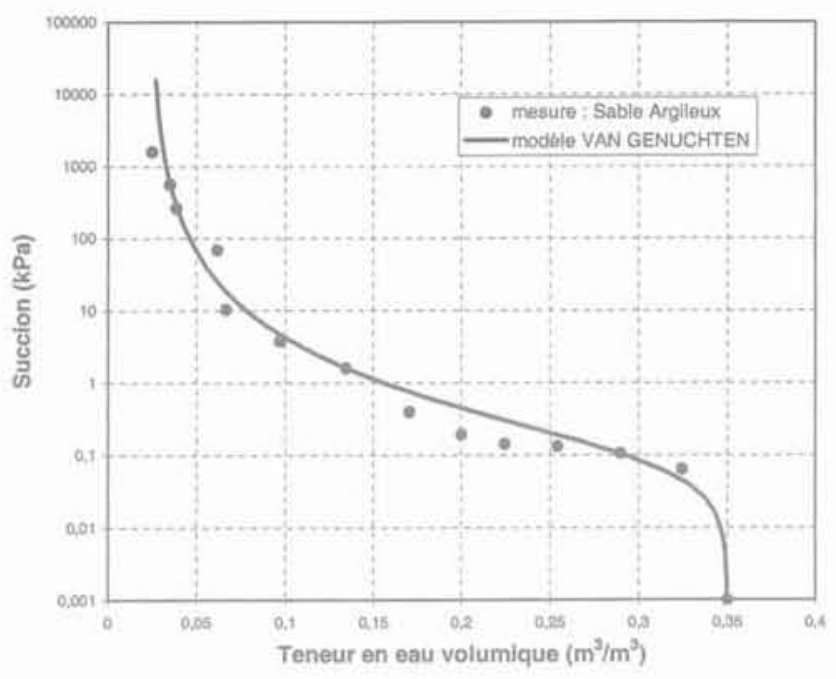

FG. 1 Courbe de rétention du sol. Soll water characteristic curve.

Les valeurs des paramètres du modèle obtenues à partir des points expérimentaux sont données dans le tableau III.

\begin{tabular}{|c|c|c|c|c|c|}
\hline \multirow[t]{2}{*}{ TABLEAU III } & \multicolumn{5}{|c|}{$\begin{array}{l}\text { Paramètres de Van Genuchten. } \\
\text { Van Genuchten parameters. }\end{array}$} \\
\hline & $\theta_{\text {, }}$ & $\theta_{2}$ & $\alpha\left(\mathrm{cm}^{-1}\right)$ & $\mathrm{m}$ & $\pi$ \\
\hline Sable argileux & 0,024 & 0,35 & 0,01 & 0,28 & 1,388 \\
\hline 3.2 & & & & & \\
\hline
\end{tabular}

La méthode TDR est basée sur la mesure du temps de parcours ( $\mathrm{t}$ ) et de l'atténuation de l'amplitude d'une onde électromagnétique envoyée le long d'une ligne de transmission (TL). Cette ligne de transmission représente la sonde de mesure. Le principe de la mesure est développé par Topp et al. (1980), qui ont montré que pour plusieurs types de sols, il existe une relation générale empirique $\theta=f(\varepsilon)$ qui relie la teneur en eau volumique $\theta\left(\mathrm{m}^{3} / \mathrm{m}^{3}\right)$ et la constante diélectrique du sol $(\varepsilon)$ et qui est de la forme:

$$
\theta=-0,053+0,029 \varepsilon-5,5 \cdot 10^{-4} \varepsilon^{2}+4,3.10^{-6} \varepsilon^{3}
$$

La constante diélectrique est obtenue à partir du temps de transit ( $t$ ) d'une onde électromagnétique à travers le sol, le long de la sonde de longueur L; soit:

$$
\varepsilon=\left[\frac{c \cdot t}{2 L}\right]^{2}
$$

où $\mathrm{c}(\mathrm{m} / \mathrm{s})$ est la vitesse de la lumière dans le vide.

Dans les appareils commerciaux, le terme [ct/2] est attribué à la longueur apparente de la ligne de transmission et noté 1 . Le rapport entre l'amplitude du signal réfléchi et l'amplitude du signal incident est mesuré comme la réflexion verticale, appelée coefficient du voltage réfléchi (ou coefficient de réflexion). Il est utilisé pour déterminer l'impédance de la ligne de transmission. Ce coefficient est défini par:

$$
\rho=\frac{R_{L}-Z_{0}}{R_{L}+Z_{0}}
$$


où $\mathrm{Z}_{0}$ est l'impédance du câble de liaison et égal à $50 \Omega$ et $\mathrm{R}_{1}$ est l'impédance du sol. La mesure de l'amplitude du signal pour $t \rightarrow \infty$ donne la valeur de $\rho$. La valeur de $R_{1}$ peut donc être calculée à partir de l'équation (25). La conductivité électrique du sol est calculée par l'intermédiaire de la constante géométrique $\mathrm{K}$ de la sonde. Cette constante géométrique est déterminée en immergeant la sonde dans une solution de conductivité électrique $\sigma_{\mathrm{a}}$ connue. On écrit alors (Nadler et al., 1991)

$$
K_{c}=\sigma_{t}\left(25^{\circ} \mathrm{C}\right) \cdot R_{2} / f_{t}
$$

où $R$, est limmédance de la solution donnée par la mesure TDR et $f$, le coefficient de correction de la température.

La conductivité électrique du sol imprégné de la solution est aussi donnée par l'équation (26). Dans ce cas, l'impédance $\mathrm{R}$, est l'ímpédance du sol obtenue par la méthode TDR.

Les sondes utilisées sont à trois tiges parallèles de longueur $80 \mathrm{~mm}$ et de diamètre $3 \mathrm{~mm}$ avec une longueur de câble de $2,50 \mathrm{~m}$. Le signal émis par un testeur de câble (Tektronix (OR) 1502B) est récupéré à l'aide d'un logiciel d'acquisition sur un ordinateur.

\section{3}

\section{Étalonnage de la méthode TDR: constante diélectrique}

Afin d'améliorer la précision des mesures TDR au cours des essaís d'infiltration, des mesures simultanées de la teneur en eau volumique $\theta$ et de la constante diélectrique $\varepsilon$ ont été réalisées sur des échantillons de sol. Les essais d'étalonnage ont été effectués pour des teneurs en eau massiques allant, généralement de 0 à $17 \%$ pour le sable argileux de la carrière de la CASEMA (BourgAchard), de 0 à $22 \%$ pour le limon de Givors et de 0 à $28 \%$ pour l'argile du Gault. On a aussi utilisé trois types de bentonites avec des teneurs en eau massiques comprises entre 26 et $195 \%$. Au cours des essais d'étalonnage des sondes, de l'eau déminéralisée et trois lixiviats (de mâchefer, de scories de plomb et de Refiom) ont été utilisés.

Afin de se conformer aux études de Topp et al. (1980), Roth et al. (1990), Nadler et al. (1991), Jacobsen et Schjonning (1993), Weiller et al. (1998), les points expérimentaux formès par les couples $(\theta, \varepsilon)$, ont été utilisés pour établir une nouvelle relation polynomiale de $3^{e}$ degré représentant la courbe d'étalonnage des sondes TDR. La nouvelle relation avec le sable argileux et le lixiviat de scorie est

$$
\theta=-0,0428+0,0324 \varepsilon-0,0015 \varepsilon^{2}+4.10^{-5} \varepsilon^{3}
$$

La fiqure 2 donne une représentation de cette loi avec tous les poirts expérimentaux utilisés. La courbe en pointillé représente les valeurs obtenues avec la relation de Topp et al. Les points expérimentaux s'écartent de la relation de Topp dès qu'on dẻpasse une teneur en volumique de $10 \%$. La nouvelle relation sera dorénavant utilisée pour le calcul de la teneur en eau volumique du sol, une fois la constante diélectrique déterminée à partir du signal TDR.

Meddahi et al. (1993) ont montré qu'il était possible de déterminer la courbe d'élution (courbe de variation de la concentration en fonction du volume sorti) d'un traceur non réactif en utilisant la méthode TDR. En effet en admettant qu'il existe une relation linéaire entre la conductivité électrique apparente d'une couche de sol d'épaisseur L et la concentration C en soluté de l'eau interstitielle (Kachanoski et al., 1992), on peut écrire:

$$
\sigma_{1}=\alpha \cdot C+\beta
$$

où $\alpha$ et $\beta$ sont deux constantes empiriques.

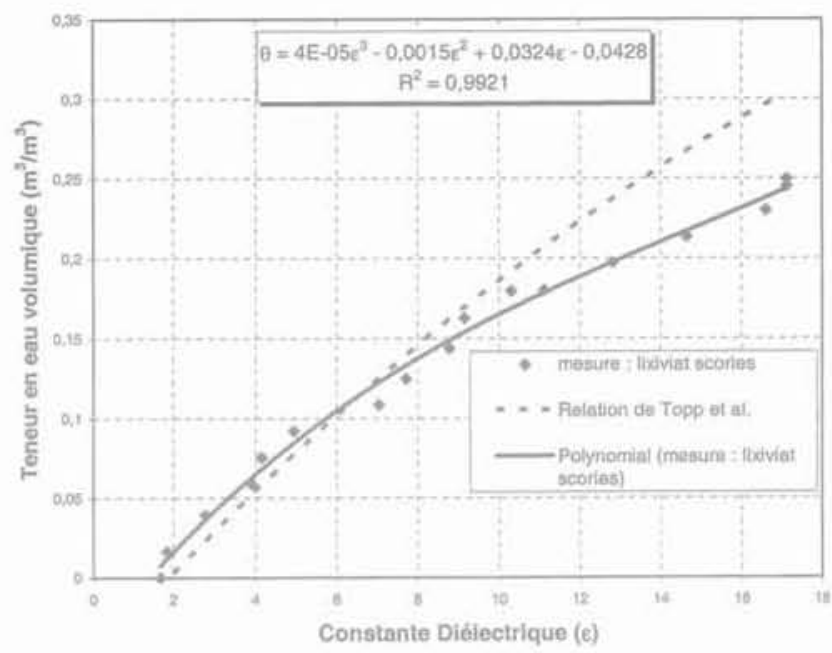

1G:2 Teneur en eau volumique en fonction de la contante diélectrique.

Volumetric water content versus dielectric constant.

Si $M_{1}$ désigne la masse de soluté par unité de surface dans la couche d'épaisseur $L$, on peut écrire

$$
\mathrm{M}_{\mathrm{L}}=\theta \mathrm{LC}
$$

$\theta$ étant la teneur en eau volumique de la couche.

Si $M_{t i 1}$ est la masse totale par unité de surface du traceur dans l'épaisseur L avant une nouvelle injection et $M_{L_{10}}$ la masse totale par unité de surface après une injection à l'instant to l'apport en masse de soluté dans la couche est donné par:

$$
M_{L, t_{0}}-M_{L, t_{5}}=\left(\sigma_{L, L_{5}}-\sigma_{L, 5}\right)\left(\frac{\theta L}{\alpha}\right)
$$

$\sigma$, est la conductivité électrique apparente de la couche à l'instant $t$ et $\sigma$, la conductivité électrique apparente après l'injection à $t_{i j}$. Si le sol est totalement lessivé à l'instant initial, $\mathrm{M}_{1+1}=0$. A un instant $\mathrm{t}>\mathrm{t}_{\mathrm{r}}$, la conductivité électrique apparente évolue dans la couche pendant la propagation du traceur. La masse totale du traceur s'écrit alors:

$$
M_{t, t}=\left(\sigma_{L+}-\sigma_{i, t}\right)\left(\frac{\theta L}{\alpha}\right)
$$

La masse relative de traceur dans la couche à l'instant test:

$$
M_{L A}(t)=\frac{M_{L t}}{M_{L, b}}=\left[\frac{\sigma_{L t}-\sigma_{L t}}{\sigma_{L, 0}-\sigma_{L, b}}\right]
$$

A partir de la relation (26) on exprime les conductivités électriques $\sigma_{1}$, du sol en fonction des impédances $R_{1 \text {. }}$ En introduisant les expressions obtenues dans la relation (32), la masse relative de soluté retenue dans la couche à l'instant t, sur l'épaisseur L, s'écrit:

$$
M_{L, A}(t)=\left[\frac{R_{L t}^{-1}-R_{L, t}^{-1}}{R_{L_{t}}^{-1}-R_{L, t_{t}}^{-1}}\right]
$$

La relation [33] permet la détermination de la masse relative de soluté résident dans le sol par la mesure de l'impédance initiale du sol $R_{1}$, de l'impédance minimum du sol $R_{t}$ et de l'impédance a l'instant $t R_{t}$ 


\section{Étalonnage de la méthode TDR: fraction soluble}

Du fait de la complexité chimique du lixiviat de scorie infiltré, on a préféré dans ce travail se contenter en première analyse, d'une approche globale où les solutés sont caractérisés par la mesure plus simple de leur fraction soluble FS. C'est également cette fraction soluble qui affecte la conductivité électrique de la solution que mesure la sonde TDR.

La détermination de la fraction soluble FS du lixiviat est obtenue par la pesée de $25 \mathrm{~cm}^{3}$ de lixiviat dans un bécher de $50 \mathrm{~cm}^{3}$ avant et après une évaporation complète du liquide dans l'étuve à $105^{\circ} \mathrm{C}$. Des mesures de conductivité électrique o par la méthode IDR et de la fraction soluble sont réalisées sur des dilutions du lixiviat de scorie. Les valeurs des fractions solubles des dilutions et leurs conductivités électriques sont rapportées aux valeurs initiales pour obtenir les paramètres relatifs FS/FS (FS, et $\sigma_{0}$ étant les valeurs initiales). Les points expérimentaux représentés sur la figure 3 par les couples $\left(\sigma / \sigma_{0} F S / F S_{0}\right)$, sont utilisés pour déterminer la corrélation entre la conductivité électrique et la fraction soluble. La relation polynomiale de $4^{e}$ degré permet lajustement des points avec un bon coefficient de corrélation: $\mathrm{R}^{2}=0,9745$.

$$
\begin{gathered}
\text { FS }_{\text {FSS }}=9,4223 \sigma / \sigma_{0}{ }^{4}-17,047 \sigma / \sigma_{0}{ }^{3}+ \\
9,5933 \sigma / \sigma_{0}{ }^{2}-0,8566 \sigma / \sigma_{0}+0,0454
\end{gathered}
$$

Cette relation permet la détermination des profils de fraction soluble au cours de l'infiltration par la mesure de la condu ctivité électrique cu sol.

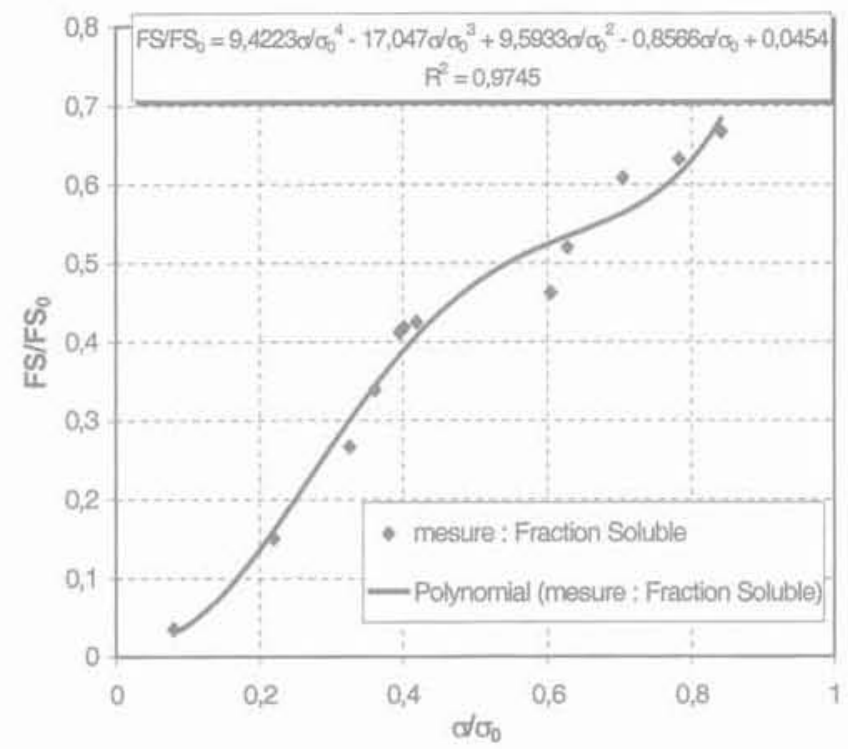

FiG.3 Relation entre la conductivité électrique relative et la fraction soluble relative. Relationship between the scaled solute concentration and the scaled electrical conductivity.

\section{Études expérimentales}

Les études expérimentales ont pour but la description de la migration des polluants du lixiviat de scories dans le sol non saturé. Des essais de sorption sont réa-

lisés sur des échantillons du sable argileux pour mettre en évidence la cinétique d'adsorption et caractériser les isothermes d'adsorption. Des essais d'infiltration sont réalisés sur des colonnes de sable argileux de BourgAchard (sols 1 et 2) sous faibles charges hydrauliques pour limiter la vitesse d'écoulement. La théorie de la convection-dispersion sera appliquée aux résultats pour évaluer les paramètres qui régissent la migration des polluants.

\section{1}

\section{Étude du phénomène de sorption du sable argileux}

Le principe de détermination de la cinétique sorption consiste à mettre une certaine masse $\mathrm{M}_{\text {de }}$ sol sec dans un volume $V$ de la solution de concentration initiale $C_{0}$ puis de mesurer la variation de cette concentration en ions au cours du temps par prélèvement et titrage. Le prélèvement et le titrage sont réalisés jusqu'à atteindre l'équilibre de sorption à la température ambiante de la pièce climatisée. La masse initiale $\mathrm{C}_{0} \times \mathrm{V}$ grammes de l'espèce chimique considérée mise en contact avec la masse $M$, de sol sec devient au temps t $C \times V$ gramme. Donc la quantité $S$, adsorbée par unité de masse sèche est:

$$
S=\frac{\left(C_{0}-C_{i}\right) V}{M_{\mathrm{s}}}(\mathrm{mg} / \mathrm{kg} \text { de sol sec) }
$$

Cette procédure de l'essai de cinétique de sorption est celle utilisée par Garcia Delgado (1996).

Comme on l'a dit plus haut, on considère ici globalement la fraction soluble. La figure 4 représente la variation temporelle de la concentration relative de la fraction soluble lors de l'essai de sorption. Sur cette figure, on remarque que la concentration relative en soluté diminue rapidement pour atteindre une valeur relative de 0,85 au bout de 10 minutes. Il s'agit donc d'une cinétique non instantanée. Au-delà de cette durée, la concentration relative reste quasi constante autour d'une valeur de 0,83. Cette quasi-stabilité montre que l'équilibre des échanges entre les ions de la solution et la matrice solide est pratiquement atteint.

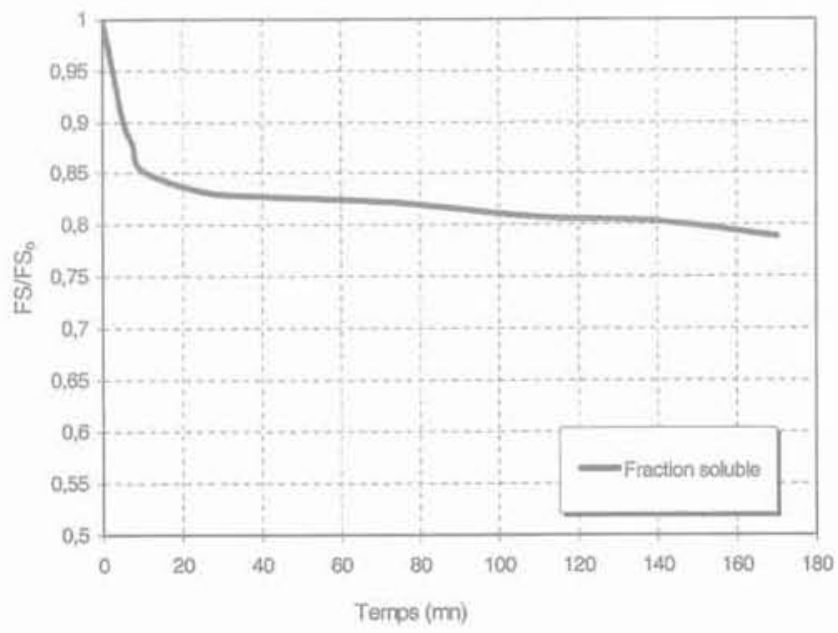

FIG.4 Cinétique d'adsorption de la fraction soluble.

Solute sorption cinetic. 
L'essai de cinétique d'adsorption est réalisé pour plusieurs valeurs de concentration initiale $\mathrm{C}_{0}$ selon la méthode utilisée par Rowe et al. (1988) et Garcia Delgado (1996). La figure 5 représente la variation de la concentration d'équilibre dans la solution en fonction de la concentration initiale en fraction soluble. Les points expérimentaux dans cette représentation sont bien alignés et peuvent être représentés par une droite de régression avec un coefficient de corrélation de 0,99. Les isothermes de sorption peuvent donc être interprétés par le modèle linéaire de Freundlich. La pente de cette droite de régression est utilisée pour le calcul du coefficient de distribution $k_{\text {. }}$ La valeur de ce coefficient pour la fraction soluble est relativement faible: $k_{\mathrm{d}}=0,345 \mathrm{ml} / \mathrm{g}$.

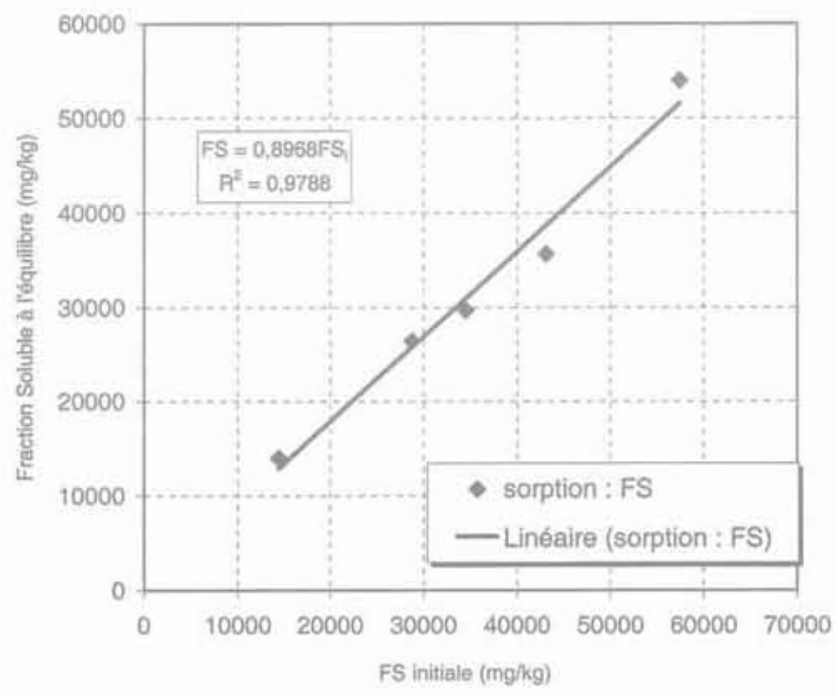

FG.5 Relation entre la concentration d'équilibre et la concentration initiale.

Relationship between the solute equilibrium concentration and initial concentration.

\section{Étude de l'infiltration du lixiviat de scories}

\section{Appareillage d'essais d'infiltration}

L'essai d'infiltration est réalisé sur une colonne de sable argileux. Cette colonne d'une hauteur de $550 \mathrm{~mm}$ est composée d'anneaux de $50 \mathrm{~mm}$ de haut et de $106 \mathrm{~mm}$ de diamètre. Le sol mouillé à la teneur en eau désirée est compacté dans chaque anneau à une densité sèche fixée (Alimi et Gaidi, 1999), La densité réelle de compactage est déterminée à la fin de l'essai. Lors du compactage du sol, dix anneaux ont été équipés de sondes TDR. Le schéma de la colonne est donné sur la figure 6 .

L'infiltration est effectuée sous une charge constante $h_{0}$ de $10 \mathrm{~cm}$ appliquée à l'entrée supérieure de la colonne. La moyenne des teneurs en eau de cornpactage est $9,3 \%$ et celle des densités sèches est 1,6. La charge $h_{0}$ est donnée par la bouteille de Mariotte et le volume infiltré est régulièrement lu sur cette bouteille. L'essai d'infiltration est arrêté lorsque 1,5 fois le volume des vides du sol de la colonne est récupéré. Les mesures des sondes TDR sont enregistrées sur un ordinateur à intervalle de temps régulier, toutes les minutes au début de l'essai et toutes les 15 minutes après 3 heures d'infiltration.

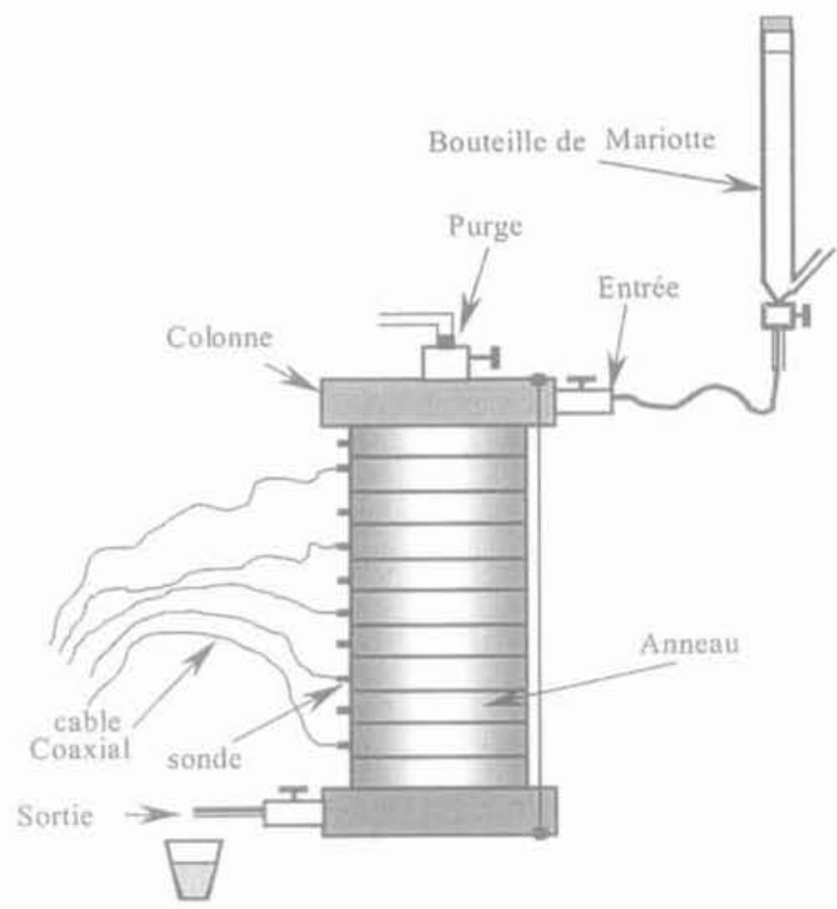

FG.6 Schẻma de la colonne d'infiltration. Column of infiltration.

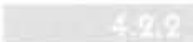

\section{Résultats des essais d'infiltration}

Les résultats des essais d'infiltration comprennent la courbe d'infiltration, les profils hydriques, les profils d'impédances et la courbe de sortie du liquide. L'analyse de ces différentes courbes permet la description du mouvement de l'eau et du soluté à l'entrée, à l'intérieur de la colonne et à la sortie. Ces courbes seront associées à des modèles théoriques pour la détermination des paramètres de ces modèles et pour apprécier leur domaine de validité.

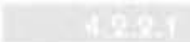

Analyse de la courbe d'infiltration

Les volumes cumulés obtenus sont tracés en fonction du temps (Fig. 7). La courbe d'infiltration $(V=f(t))$ de cette figure montre qu'il s'agit bien d'une infiltration régulière, caractérisêe par une montée rapide au début

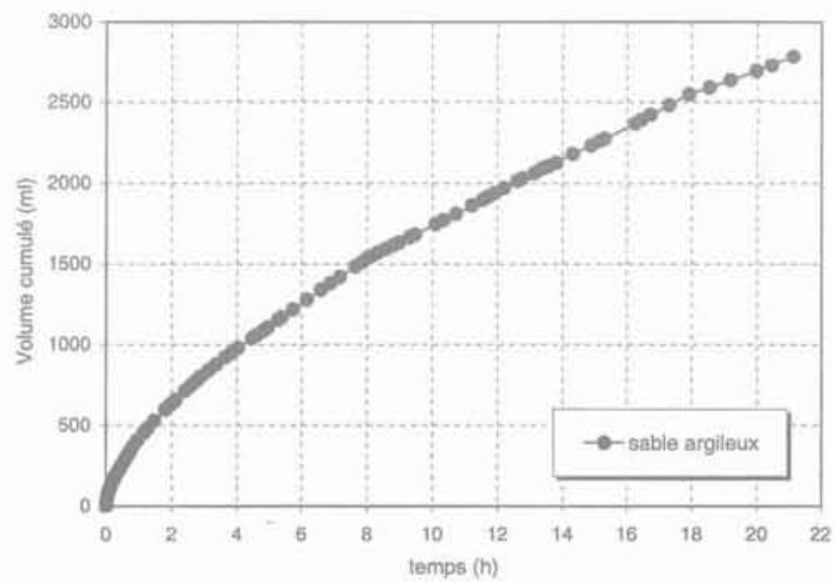

FG.7 Courbe d'infiltration. Infiltration curve. 
de l'essai. Cette rapidité résulte de la forte succion du sol dans son état initial (Alimi et Gaidi, 1999); on observe ensuite une progression linéaire de l'infiltration avec le temps. Le régime permanent est atteint au bout de 5 heures d'infiltration.

L'infiltration cumulée est calculèe à partir du volume $\mathrm{V}$ infiltré à l'instant t par: $\mathrm{I}=\mathrm{V} / \mathrm{A}$ (A étant la section de la colonne de sol). Sur la figure 8 les valeurs de l'infiltration cumulée sont représentées en fonction de $(\sqrt{t})$. Dans le diagramme $(\sqrt{t} ; 1)$ le modèle de Philip représenté par l'équation (6) est une droite dont la pente est la sorptivité du sol. On remarque que les points d'infiltration peuvent être représentés par une demi-droite jusqu'à une durée de 30 minutes avec un bon coefficient de corrélation R (au-dessus de $98 \%$ ). Aư-delà de cette durée, la courbe d'infiltration ne peut plus être représentée par ce modèle. D'après la théorie de l'infiltration, l'écart entre les points expérimentaux et la relation (6) provient de l'influence de la dimension de l'échantillon et surtout de celle de la gravité sur l'écoulement puisque la quantité infiltrée est plus importante que celle prédite par le modèle. Néanmoins, on peut déduire de la valeur de la sorptivité, la diffusivité du lixiviat dans le sol au début de l'essai. On constate que la diffusivité du lixiviat $\left(\mathrm{D}=1,65.10^{-6} \mathrm{~m}^{2} / \mathrm{s}\right.$ ) est plus élevée que celle de l'eau ( $\left.\mathrm{D}=1,6.10^{-7} \mathrm{~m}^{2} / \mathrm{s}\right)$ dans les mêmes conditions d'infiltration dans le sol étudié (Gaidi, 2002). Ce résultat prouve que le lixiviat modifie la capacité d'adsorption du sol d'où le changement de la valeur de la diffusivité. Le tableau IV présente les valeurs de la vitesse d'infiltration $v$ en régime permanent, de la vitesse d'infiltration initiale $v_{\text {, }}$ de la vitesse à la sortie de la colonne $v_{\text {, }}$, de la sorptivite $S\left(\theta_{0}, \theta_{0}\right)$ et de la diffusivité $\mathrm{D}$. On remarque que la vitesse initiale aux premiers instants de l'infiltration est 10 fois plus élevée que la vitesse d'infiltration en régime permanent et que la vitesse de sortie du percolat. La vitesse d'infiltration peut donc surestimer le coefficient de perméabilité d'un sol si la durée de l'essai n'est pas suffisante.

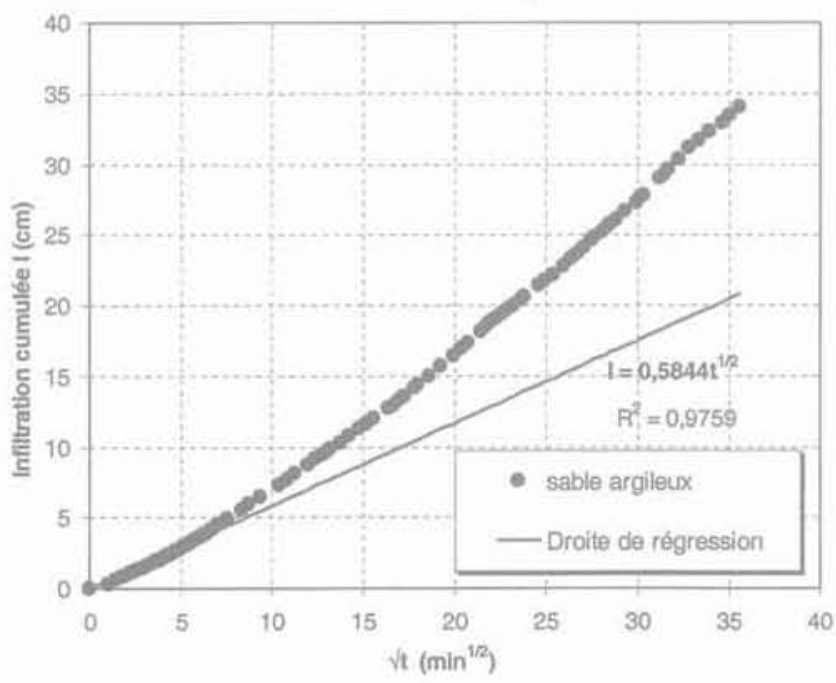

FG. 8 Courbe d'infiltration en racine carrée du temps.

Infiltration curve with square root of time.

\begin{tabular}{|c|c|c|c|c|}
\hline TABLEAUI & $\begin{array}{l}\text { Par: } \\
\text { Soil }\end{array}$ & $\begin{array}{l}\text { tres hy } \\
\text { odynan }\end{array}$ & $\begin{array}{l}\text { Irodynamiques d } \\
\text { c parameters. }\end{array}$ & u sol. \\
\hline $\begin{array}{l}\text { Vitesse } \\
y_{h}(\mathrm{~m} / \mathrm{s})\end{array}$ & $\begin{array}{l}\text { Vitesse } \\
\mathrm{v}(\mathrm{m} / \mathrm{s})\end{array}$ & $\begin{array}{l}\text { Vitesse } \\
\mathrm{v}(\mathrm{m} / \mathrm{s})\end{array}$ & $\begin{array}{l}\text { Sorptivité } S\left(\theta_{0}, \theta_{\mathrm{\rho}}\right) \\
\left(\mathrm{em} / \mathrm{s}^{1 / 2}\right)\end{array}$ & $\begin{array}{l}\text { Diffusivité } \\
\text { D }\left(\mathrm{m}^{2} / \mathrm{s}\right)\end{array}$ \\
\hline
\end{tabular}

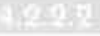

Analyses des profils hydriques et de l'impédance dans la colonne de sol

On présente sur la figure 9 les profils hydriques au cours de l'infiltration du lixiviat de scories. A l'instant $t$ = 10 minutes, l'augmentation de la teneur en eau volumique à la profondeur $12,5 \mathrm{~cm}$ signifie que l'humidité a atteint cette profondeur. A l'instant $t=1,5$ heure, la teneur en eau en bas de la colonne (sonde 10) commence à croitre. Le profil hydrique à l'instant $t=$ 2,67 heures correspond au début de la sortie du liquide du bas de la colonne de sol. Pendant que le lixiviat percole à travers la colonne, les profils hydriques continuent à évoluer. Cette évolution montre que le sol continue à retenir une partie du liquide infiltré. A partir de 4,17 heures, le sol a atteint sa teneur en eau volumique d'équilibre. Cette teneur en eau d'équilibre est proche $0,36 \mathrm{~m}^{3} / \mathrm{m}^{3}$. Cette valeur représente $90 \%$ de la porosité du sol compacté à une densité sèche $\gamma_{\mathrm{d}} / \gamma_{\mathrm{h}}$ égale à 1,6. On obtient alors une distribution uniforme des teneurs en eau dans la colonne de sol. Cette allure du profil hydrique montre que l'infiltration du lixiviat n'entraine plus une augmentation de la teneur en eau: toute la quantité d'eau infiltrée est relâchée en quantité analogue. La valeur de cette teneur en eau est en accord avec la valeur donnée par le modèle de Van Genuchten lorsque la succion est nulle. Apparemment la présence de soluté dans l'eau ne modifie pas la courbe de rétention de l'eau au cours de l'imbibition.

Teneur en eau volumique $(\mathrm{m} 3 / \mathrm{m} 3)$

$\begin{array}{lllllllllllll}0 & 0.05 & 0.1 & 0.15 & 0.2 & 0.25 & 0.3 & 0.35 & 0.4 & 0.45 & 0.5 & 0.55 & 0.6\end{array}$

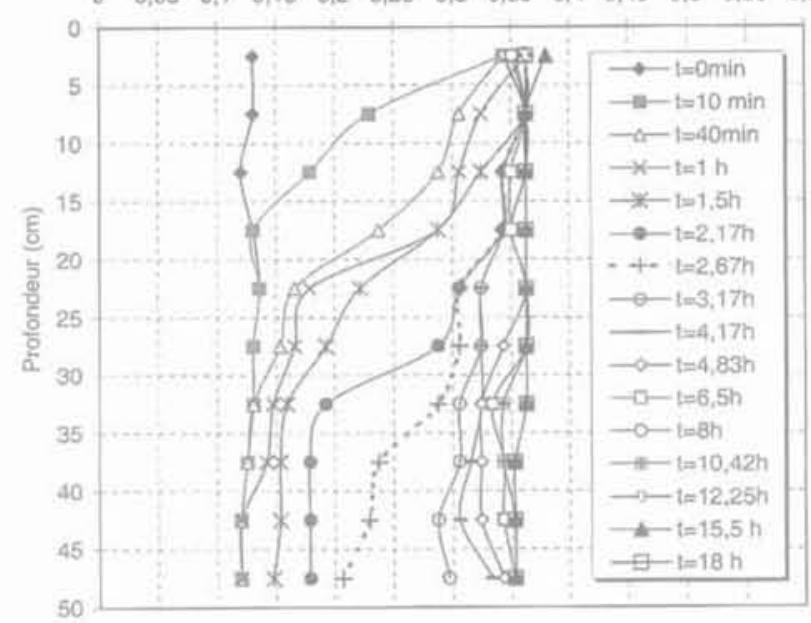

FIG,9 Profils hydriques - lixiviat de scories. Moisture profiles - lead slag leachate.

Nous avons également tracé les courbes de variation spatio-temporelle de l'impédance lors de l'infiltration. La diminution de l'impédance du sol correspond à l'augmentation de la quantité d'ions en solution dans le liquide interstitiel (Fig. 10). Après 40 minutes d'infiltration, les profils d'impédance sont moins étalés que les profils hydriques. Il y a donc une accumulation d'ions plus importante dans la partie supérieure de la colonne. Après 1,5 heure d'infiltration, quand l'humidité atteint le bas de la colonne, les profils d'impédance deviennent aussi étalés que les profils hydriques sur les 40 premiers centimètres. Il n'y a plus de retard entre le mouvement de l'humidité et celui des polluants en solution. Au début de la sortie du lixiviat de la colonne de sol, l'impédance du sol des 10 premiers centimètres ne 
varie plus tandis que celle du sol des 10 derniers centimètres continue à diminuer. La concentration en ions de l'eau interstitielle des 10 derniers centimètres continue à augmenter.

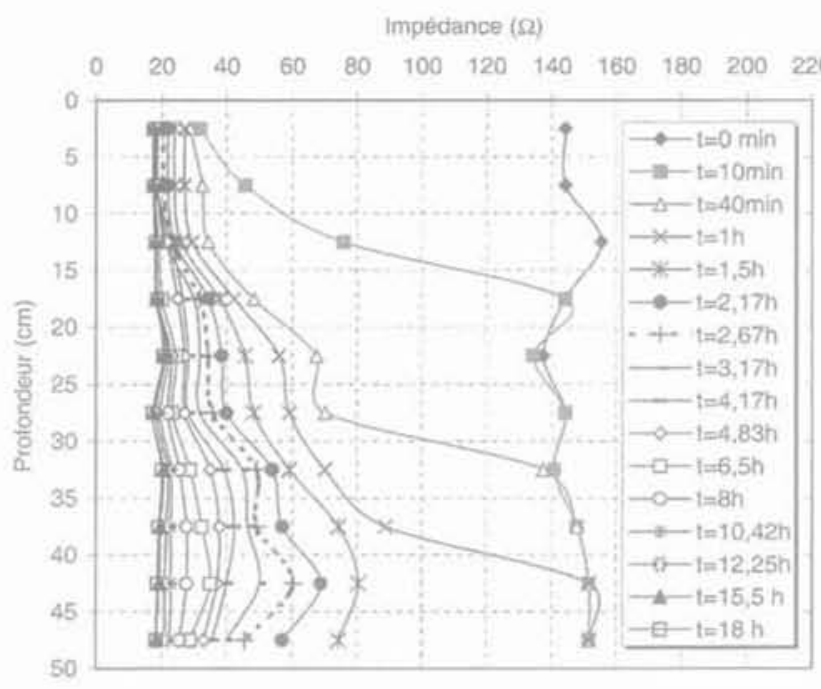

FG. 10 Profils des impédances. Impedance profiles.

Les profils d'impédance de la figure 10 permettent la détermination de l'impédance initiale du sol $R_{\text {, }}$, de l'impédance minimum du sol $R_{L L}$ et l'impédance à l'instant $t R_{L}$ à une profondeur $L$ donnée. On peut donc calculer la masse relative de soluté retenue à l'instant $t$, au-dessus de la profondeur L à l'aide de la relation (33) (Meddahi et al., 1993). L'étude de l'évolution de cette masse relative dans le temps permet la détermination du temps nécessaire pour la saturation en soluté de l'épaisseur L du sol. C'est le temps nécessaire pour que le flux de polluant qui arrive à la profondeur L la traverse entièrement. La valeur de ce temps est un paramètre d'appréciation de l'efficacité d'une barrière étanche d'épaisseur L.

L'évolution de masse relative de soluté retenue est. représentée sur la figure 11 pour les profondeurs 125, 275 et $475 \mathrm{~mm}$. On remarque qu'effectivement la pollution atteint le bas de la colonne après une heure

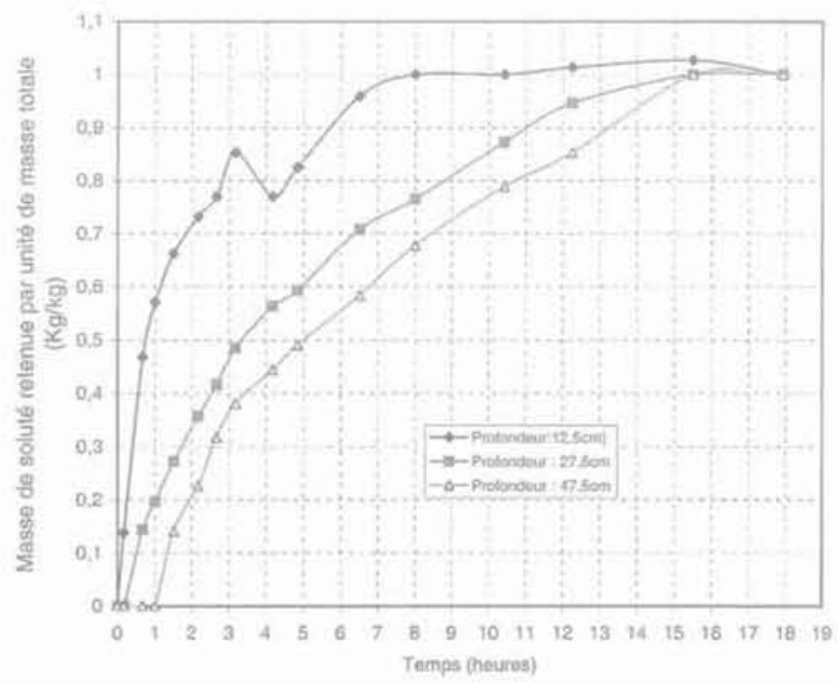

FG. 11 Évolution de la masse de soluté retenue dans le sol sur une profondeur donnée. Retained pollutant change at different soil depths. d'infiltration. La capacité de rétention des 12 premiers centimètres est atteinte au bout de 8 heures alors qu'i] faut deux fois plus temps pour atteindre la limite de rétention du sol sur la profondeur de $47 \mathrm{~cm}$. Ce résultat montre que l'épaisseur d'une couche participe à la limitation de la transmission des polluants au milieu récepteur. On ne considère actuellement que la valeur de la perméabilité du sol pour le choix des matériaux d'étanchéité. Le temps de transfert augmente quand l'épaisseur de la couche augmente. On remarque que le sol continue à jouer le rôle d'un filtre 15 heures après la sortie du liquide de la colonne. Les mesures TDR permettent la mise en évidence du róle complexe d'une couche d'étanchéité.

\section{5}

\section{Étude du mode de migration des polluants dans le sol}

La connaissance du mode de migration des polluants dans les sols permet la mise au point d'un bon outil de prévision du flux transféré à travers une couche. Les modèles de transfert par convection ou par convection-dispersion sont utilisés pour le transfert des solutés dans les sols saturés. Mais lorsque le transfert a lieu dans un sol non saturé, différents phénomènes de partage apparaissent, partage de l'eau entre phase mobile et phase immobile, partage des solutes par sorption-désorption, et influencent le mode de migration. Pour décrire les modes de transfert des solutés au cours de la saturation on analyse les profils de concentration.

Le volume du percolat recueilli au cours du temps est utilisé pour le calcul du coefficient de perméabilité. Ce coefficient de perméabilité est comparé à la vitesse d'infiltration et à la vitesse de convection du soluté (vitesse de pore). On peut alors relever les écarts entre les paramètres hydrodynamiques déterminés à partir des courbes d'entrée du liquide dans le sol et les paramètres du mouvement du liquide dans le sol.

La solution (18) de l'équation de transfert de soluté pour une profondeur égale à la longueur de la colonne, donne l'évolution de la concentration en soluté à la sortie de la colonne et permet l'estimation du coefficient de dispersion. Ce coefficient de dispersion peut être comparé à celui obtenu à l'aide des profils de concentration.

\section{1}

\section{Analyse des profils de concentration}

La relation (34) est utilisée pour le calcul de la fraction soluble à partir des mesures de la conductivité électrique du sol. Les profils de fraction soluble relative FS/FS obtenus par ces calculs, sont représentés sur la figure 12. Durant les trois premières heures d'infiltration, les valeurs de la fraction soluble augmentent principalement dans la partie supérieure même après la sortie du lixiviat de la colonne de sol. La concentration de soluté dans le liquide interstitiel est constante sur les 12 premières centimètres mais change au cours du temps. L'allure des profils de fraction soluble relative montre que la migration des polluants se fait par convection-dispersion après 40 minutes d'infiltration. Dès la sortie du lixiviat de la colonne de sol, on constate 
une accumulation des polluants dans la partie basse. Après 6 heures d'infiltration, le mouvement de convection devient prépondérant. Donc durant la saturation de la colonne de sol, la nature du mouvement de transport de polluant change. Le mouvement des polluants est purement diffusif les premières minutes d'infiltration, puis convectif-dispersif pour devenir purement convectif. La durée de chaque mode de migration doit dépendre de la nature du sol.

\section{FS/FS。}

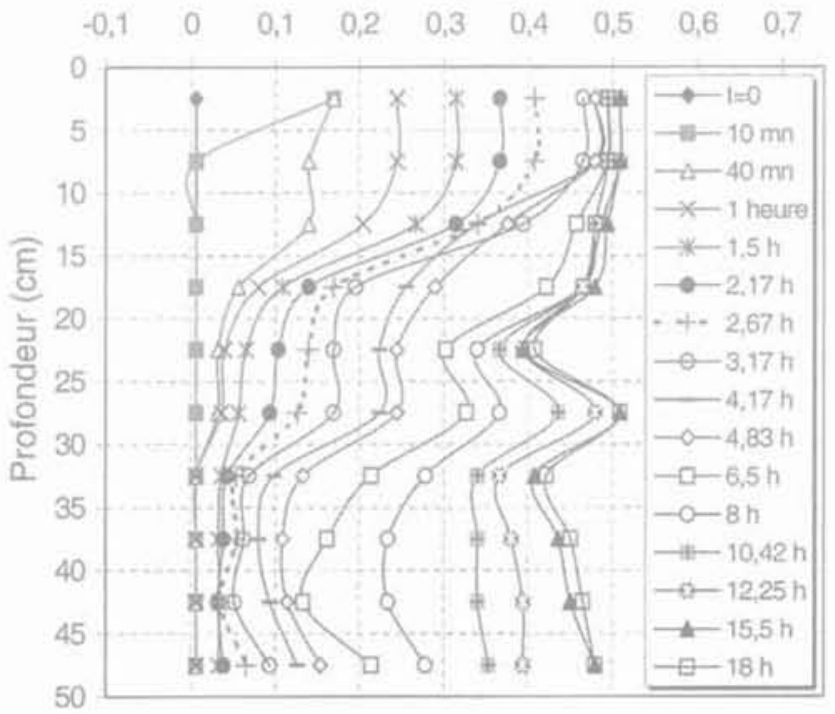

FG. 12 Profils de fractions solubles. Solute concentration profiles.

En comparant les profils hydriques (Fig. 9) aux profils de fraction soluble relative (Fig. 12), on constate qu'après 10 minutes d'infiltration, I'humidité est arrivée à la profondeur $12,5 \mathrm{~cm}$ alors que l'augmentation de la fraction soluble n'apparait que sur $2,5 \mathrm{~cm}$. Lorsque la teneur en eau d'équilibre est atteinte au bout de 4,17 heures d'infiltration, la fraction soluble du liquide interstitiel n'a pas encore atteint sa valeur d'équilibre sur toute la hauteur de la colonne de sol. Elle n'est atteinte sur toute la hauteur que 15,5 heures après le début de l'infiltration. Cette durée correspond à celle requise pour atteindre la capacité de rétention de polluant dans le sol sur la profondeur de $47,5 \mathrm{~cm}$ (Fig. 11). Pour quantifier ce retard de la migration des polluants dans le sol, on utilise les relations (10), et (21) qui donnent la profondeur mouillée z et la profondeur du soluté $\mathrm{z}$. La fonction de retard $\mathrm{R}$ est évaluée pour une isotherme linéaire $(\mathrm{N}=1)$. Les résultats de calcul sont portés sur la figure 13. On constate qu'au cours de l'infiltration, la profondeur mouillèe augmente plus vite que la profondeur du soluté. Après 4 heures d'infiltration, la profondeur mouillée est déjà égale à la hauteur de la colonne de sol alors que le polluant se concentre sur les 10 premiers centimètres. L'écart entre les deux profondeurs augmente durant ces quatre premières heures d'infiltration, durée de la saturation du sol. Pendant cette saturation, la propagation du front d'humidité et du front de soluté se fait à vitesse constante et la vitesse de propagation de l'eau est quatre fois plus élevée que celle du soluté. Ceci est en accord avec les observations faites sur les profils d'humidité et les profils de fraction soluble relative. Les modèles correspondant aux relations (10) et (21), donnent une bonne prédiction des profondeurs dans le cas du sol utilisé

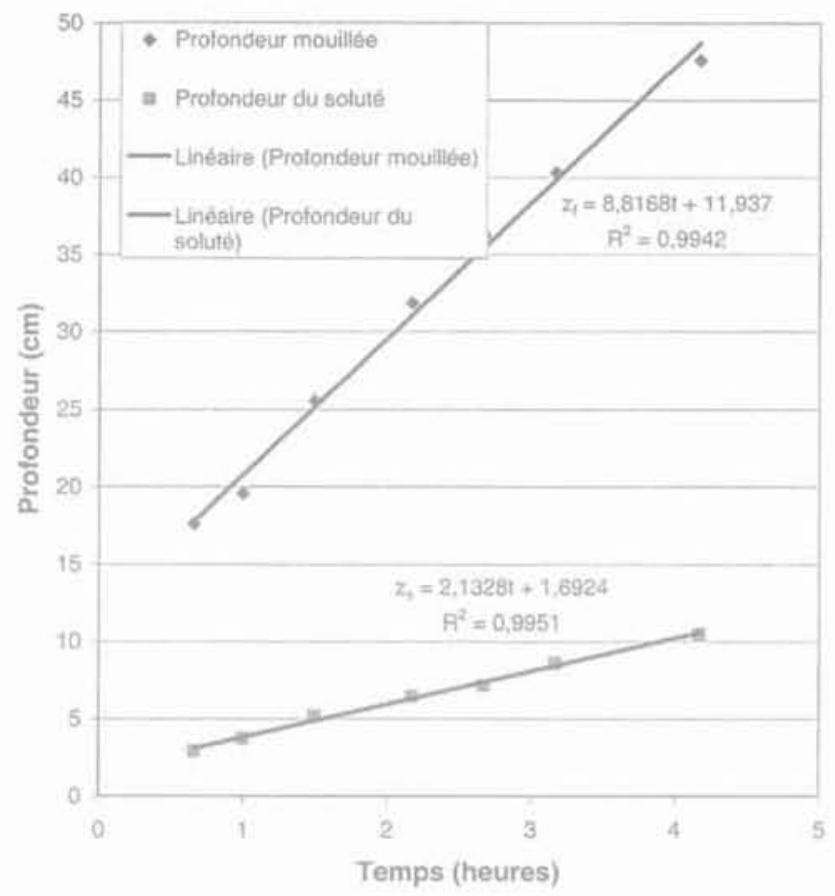

Fis. 13 Évolution comparée des fronts de Thumidité et de pollution.

Wetted front and pollution front movement.

\section{Évaluation des coefficients de dispersion de la fraction soluble}

Pour évaluer le coefficient de dispersion de soluté dans le sol au cours de l'infiltration et son évolution au cours de la saturation, on suppose que la migration se fait par convection-dispersion. On admet aussi les hypothèses de la méthode statistique en utilisant la loi de Gauss comme loi de distribution de la fraction soluble relative le long de la colonne de sol (20). L'écarttype est donné par la différence des profondeurs $z_{015}$ et $z_{\text {og }}$; soit:

$$
2 \sigma=z_{0,15}-z_{0,89}=2 \sqrt{2 D_{d} t}
$$

où $z_{0,1}$ est la profondeur correspondant à $16 \%$ de la concentration $\mathrm{C}_{0}$ et $z_{0.8}$ la profondeur correspondant à $84 \%$ de la concentration $C_{\text {. }}$. On utilise les profils de concentration relative (Fig. 12) pour l'estimation de la valeur du coefficient apparent de dispersion, au cours de la saturation. On peut tracer sa courbe d'évolution avec le temps. Lorsque le sol est réactif. $\mathrm{D}_{\text {d }}$ doit être remplacé par le rapport D/R dans l'équation (36), $\mathrm{A}$ étant le coefficient de retard. Le calcul de $R$ est fait pour une isotherme linéaire de Freundlich avec la valeur du coefficient de distribution $\mathrm{k}_{\mathrm{d}}=0,345 \mathrm{ml} / \mathrm{g}$, obtenue pour la fraction soluble. Les résultats de calcul, présentés sur la figure 14, montrent que le coefficient de dispersion évolue au cours de la saturation du sol. La loi d'évolution n'est pas bien définie, les valeurs de $D_{\text {f }}$ calculées étant très dispersées. Apparemment ce coefficient croît au cours de la saturation du sol d'essai. Les polluants se dispersent moins vite dans les zones non saturées. 


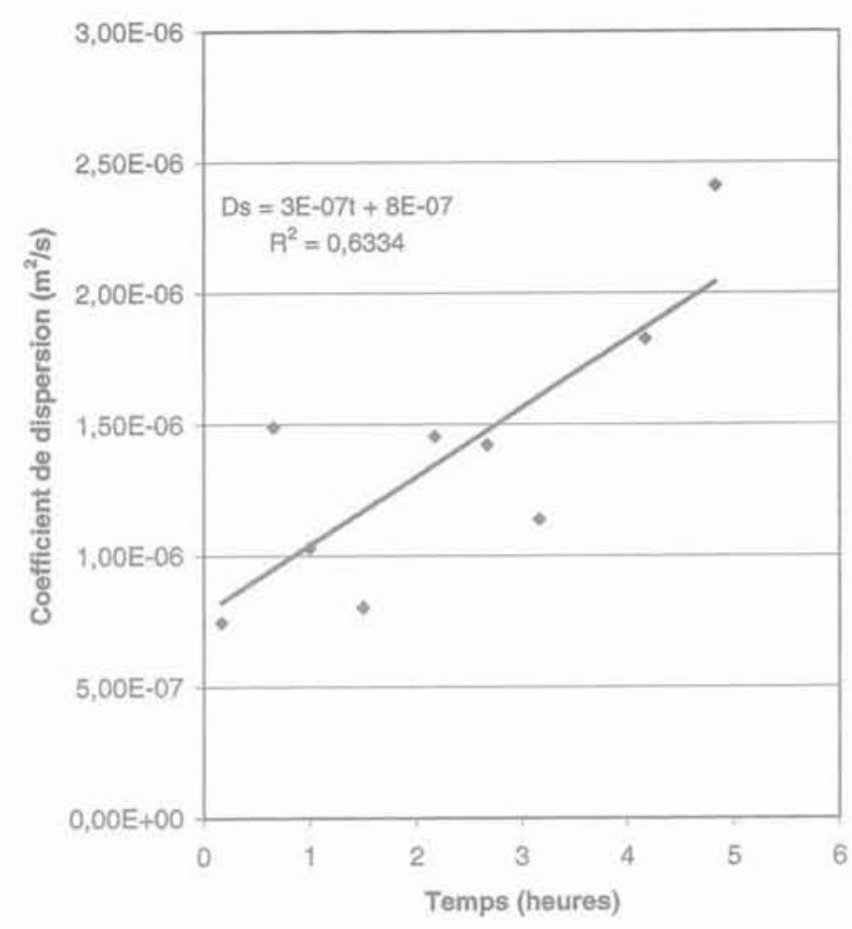

FG.14 Évolution du coefficient de dispersion apparent du soluté au cours de la saturation.

Change on the apparent dispersion coefficient during leachate infiltration.

\section{Analyse des courbes de sortie}

Une méthode de description du transport des polluants dans les sols est l'analyse de la courbe de sortie du lixiviat. Au cours de cette sortie, les valeurs du volume percolé, de la conductivité électrique et de la fraction soluble du percolat, sont relevées, Le débit du liquide sortant est utilisé pour suivre l'évolution de la perméabilité du sol au cours l'écoulement.

La figure 15 représente la variation du volume sortant en fonction du temps. Dès la première heure, la vitesse de sortie devient pratiquement constante ( $v_{\text {s }}=$ $3,410^{-6} \mathrm{~m} / \mathrm{s}$ ). Les valeur's du coefficient de perméabilité

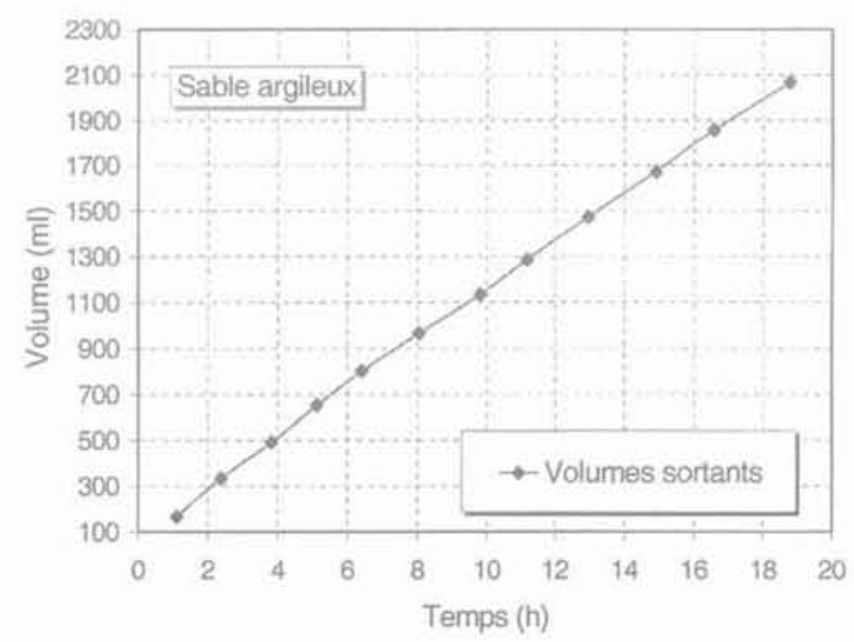

FIG. 15 Variation du volume sortant en fonction du temps.

Outflow volume variation versus time. calculées à partir des volumes récupérés prouvent que la perméabilité du sol évolue très rapidement les quatre premières heures pour ensuite atteindre lentement une valeur de $3.10^{-6} \mathrm{~m} / \mathrm{s}$ (Fig. 16). Cette valeur du coefficient est dix fois plus élevée que celle de la perméabilité à l'eau du mêrne sol dans les mêmes conditions d'essai (Alimi-Ichola et Gaidi, 1999). Ce comportement est en accord avec celui décrit par la valeur de la diffusivité du lixiviat qui correspond à une modification de la capacité de rétention du sol.

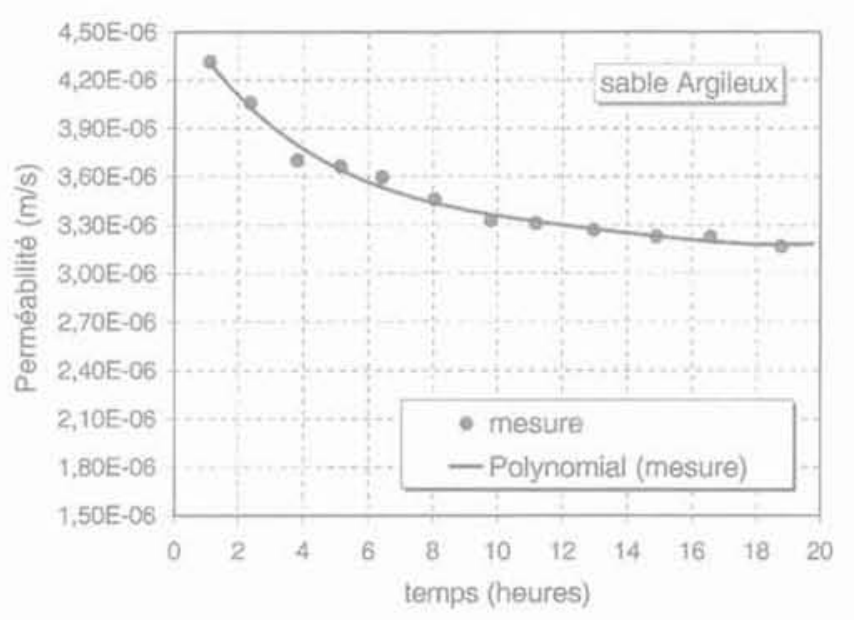

F16.16 Variation de la perméabilité à la sortie de la colonne.

Permeability coefficient variation during leachate outflow.

L'utilisation de la méthode statistique permet le calcul de la vitesse de pore à partir de la profondeur moyenne $\bar{z}=u t$. Après la détermination des profondeurs moyennes sur les profils hydriques, on calcule les valeurs de u à chaque instant $t$. En fait, cette vitesse de déplacement de la profondeur moyenne de l'humidité est égale à la vitesse de convection des polluants. Sur la figure 17 sont représentées les courbes d'évolution de la vitesse d'infiltration, la vitesse de convection et la vitesse de sortie du lixiviat, au cours du temps. On constate que la vitesse de convection est 1,5 fois plus élevée que la vitesse d'infiltration au début de l'essai. La participation de la succion du sol non saturé au mouvement de I'humidité explique cette différence. Les trois vitesses décroissent vers une valeur commune de $3.10^{-6} \mathrm{~m} / \mathrm{s}$, vitesse mesurée en régime permanent, à la sortie de la colonne. Ce résultat montre qu'un délai de plusieurs heures d'infiltration est nécessaire pour obtenir une vitesse d'infiltration qui corresponde à la perméabilité du sol.

On détermine la fraction soluble de la solution sortante au cours du temps. Les valeurs mesurées sont comparées aux valeurs de $\mathrm{FS} / \mathrm{FS}_{0}$ calculées à partir des conductivités électriques de la solution à la sortie de la colonne de sol et la relation (34). Les résultats de ce calcul sont présentés sur la figure 18. On constate un bon accord entre les valeurs calculées à partir de la relation (34) et les valeurs mesurées. Le coefficient de dispersion apparent de la fraction solution peut donc ètre déterminé à partir de la courbe d'évolution de la conductivité électrique de la solution à la sortie. Cette courbe correspond bien à la courbe de sortie des solutés.

Afin de valider l'utilisation de la relation (34) pour le calcul de la courbe d'élution et par conséquent la détermination du coefficient de dispersion apparent, les deux 


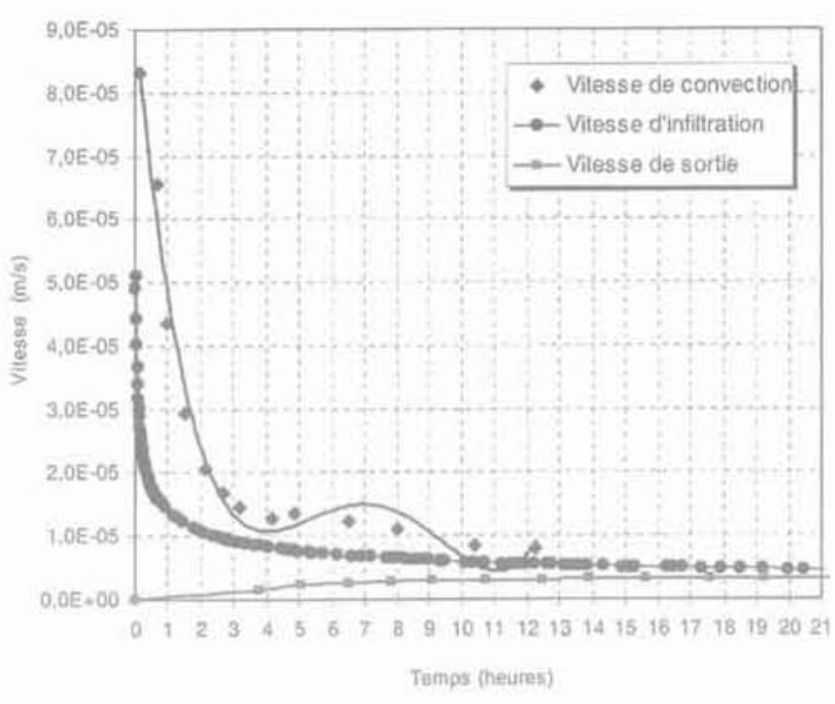

Fla.17 Évolution des différentes vitesses de mouvement du liquide dans le sol. Change with time of the different liquid motion rates.

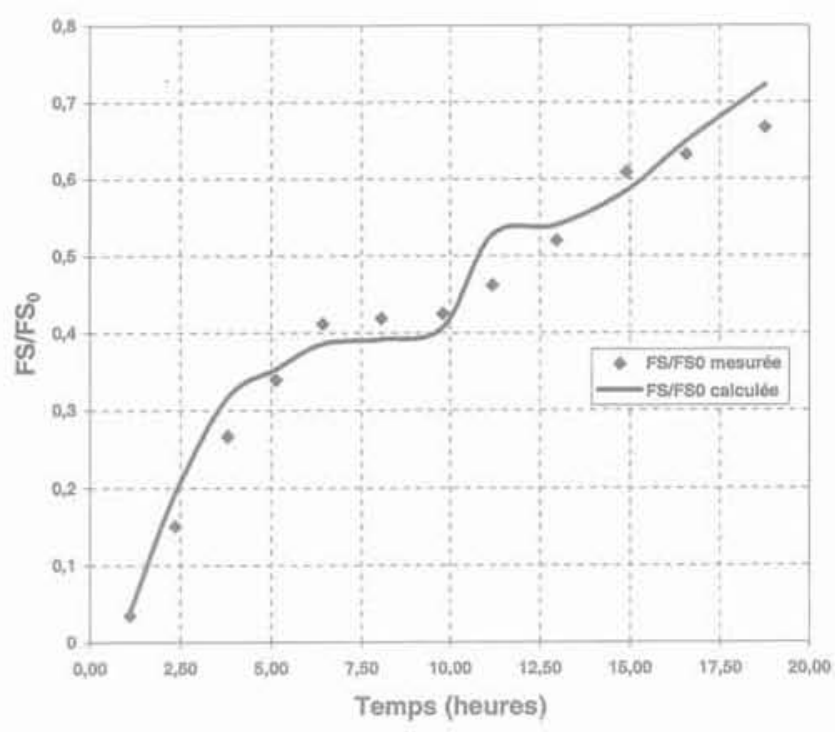

FIG. 18 Comparaison des fractions solubles mesurées et calculées.

Comparison of measured and calculated solute concentration.

séries de valeurs représentées sur la figure 18 sont utilisées. Pour ce calcul, on utilise la relation (18) dans laquelle $D_{\text {f }}$ est remplacé par D / R pour tenir compte du phénomene de retard et la variable x est remplacée par la longueur de la colonne de sol. Les valeurs du coefficient de dispersion apparent calculées à partir des deux courbes (calculée à partir de la conductivité électrique et les points de mesure), sont peu différentes les unes des autres (Fig. 19). Elles varient peu au cours du temps et sont 10 fois plus élevées que celles obtenues au cours de la saturation de la colonne (Fig. 15). Malgré la dispersion des valeurs mesurées on remarque que le coefficient de dispersion apparent du soluté dans le sol augmente pendant la saturation et atteint une valeur d'équilibre lorsque la teneur en eau d'équilibre est atteinte dans le sol. On aurait pu calculer la courbe d'élution à une profondeur quelconque z à partir de la conductivité électrique du sol et la relation (34). Ainsi en plaçant des sondes à une profondeur $\mathrm{z}$ on peut déterminer le coefficient de dispersion apparent de soluté d'une couche de sol en place.

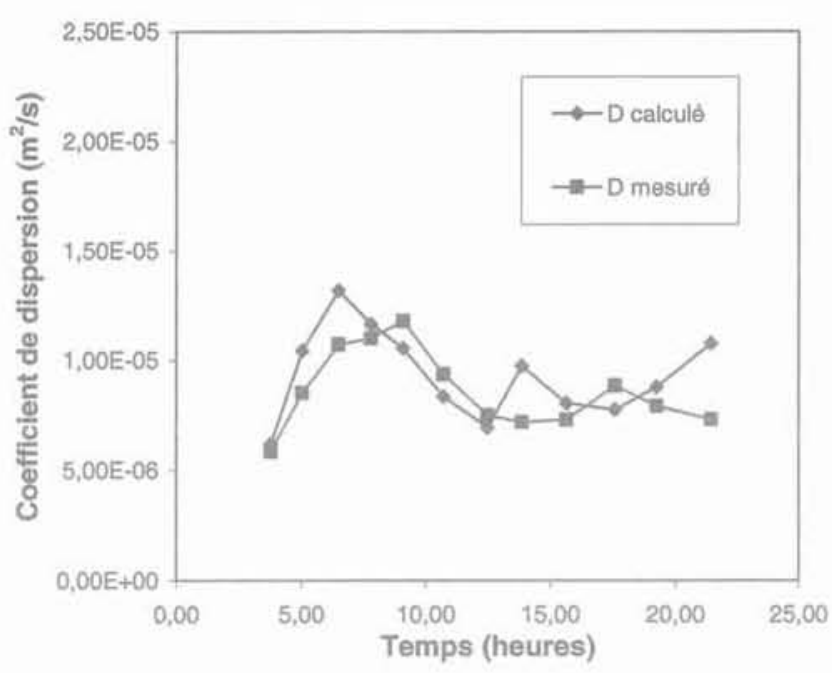

FIG, 19 Evolution du coefficient de dispersion apparent d'après la courbe d'élution.

Apparent dispersion coefficient changes during leachate outflow.

\section{6 \\ Conclusion}

Le fait de mesurer la teneur en eau et l'impédance du sol par des sondes TDR permet le suivi des polluants à l'intérieur d'une couche de sol. On peut donc utiliser une sonde TDR comme un appareil de sondage qui permet, la localisation des agents polluants et la description de leur mouvement au cours du temps.

Les mesures TDR effectuées au cours de l'infiltration permettent la description du comportement hydrodynamique du sol. On note que le liquide traverse le sol avant la saturation de la couche, que l'humidité progresse plus vite que le soluté. Ces informations ne peuvent être obtenues par les méthodes classiques qu'après le prélèvement des échantillons. Ceci suppose l'arrèt de l'infiltration. Or, un calcul correct du coefficient de perméabilité ne peut se faire qu'après la connaissance de la profondeur saturée. Cette profondeur peut varier pendant l'arrêt de l'infiltration. La comparaison des vitesses d'entrée et de sortie montre que la vitesse d'entrée de l'eau dans la colonne est supérieure à la vitesse de sortie pendant plus de dix heures. Or le régime permanent d'infiltration est atteint au bout de 5 heures. Puisque la vitesse d'infiltration décroit, le coefficient de perméabilité déterminée à partir d'un essai d'infiltration doit être supérieur au coefficient réel si la durée d'infiltration n'est pas suffisante.

Le fait d'avoir accès à la vitesse de convection donne la possibilité de réaliser une bonne description du mouvement des polluants dans la zone non saturée. On peut donc estimer le flux de polluant qui traverse cette zone à un instant donné. On a ainsi un moyen pour évaluer l'efficacité d'une barrière et pour juger de la compatibilité du flux de polluants qui traverse la barrière, avec le milieu récepteur.

La mesure de l'impédance du sol traversé par un polluant permet la définition de l'épaisseur utile si on connaît le flux acceptable par le milieu récepteur. Cette mesure peut permettre la justification des recommandations faites pour l'utilisation des barrières étanches. Au lieu de préconiser une valeur de perméabilité et une 
épaisseur, on pourra ajouter la durée nécessaire pour atteindre la capacité de rétention du polluant dans l'épaisseur préconisée. Apparemment plus l'épaisseur de la couche est grande, plus cette durée est importante.

Le coefficient de dispersion apparent augmente au cours de la saturation. Il est pratiquement multiplié par 10 lorsqu'on atteint la teneur en eau d'équilibre. Ceci explique le retard du mouvement des polluants dans le sol non saturé même si l'adsorption des éléments chimiques par les particules solides n'est pas importante.

Les différents résultats obtenus lors des essais montrent que l'on peut décrire la migration de plusieurs especes chimiques dans le sol par la fraction soluble de la solution. On peut donc faire économie d'analyses coûteuses des éléments chimiques contenus dans le lixiviat.

La détermination de la profondeur d'humidité permet un calcul plus précis du coefficient de perméabilité lors d'un essai d'infiltration in situ et donne une limite de la zone atteinte par la pollution. La détermination de la profondeur du front de pollution à partir du volume infiltré donne une idée de l'épaisseur à donner à une couche étanche. Des essaîs supplémentaires avec d'autres types de solutions et d'autres matériaux permettront de confirmer ces observations.

\section{Bibliographie}

Alimi-Ichola L, Gaidi L - Soil pollution characterisation during infiltration tests. Proceedings of $2^{\text {nd }}$ Conference orqanised by BGS, Geoervironmental Engineering, Ground contamination: pollutant management and remediation. R.N. Yong and H.R. Thomas (eds), 1999, p. 201-208.

Bentoumi O. - Transfert par infiltration de l'eau dans les sols fins compactes non satures. Etude de la diffusivité et de la conductivité hydraulique. Thèse de doctorat, INSA, Lyon, 1995.

Buckingham J.W. - Studies of movement of soll moisture. USDA. Bureau Soils Bull, $n^{\circ} 38$, USDA Washington DC, Rev. (130), Class (IB), 1907.

Childs E.C., Collis-George N. - The per. meability of porous materials. Proc. Roy. Soc, London, A-201, 1950, p. 392-405.

Elrick D.E., Robin M.J., Laryea K.B.Hydrodynamic dispersion during absorption of water by soil. 1. Model soil-moisture profiles, J. Hydrol., vol. 65. 1983, p. 313-331.

Gaidi L. - Transfert par infiltration de leaL et du soluté dans les sols non saturès. Utilisation de la méthode TDR. Thèse de cioctorat en génie civil. INSA de Lvon, 2002

Gaidi L., Allmi-Ichola L-Etudes des caractéristiques hydrodynamique des sols par la méthode TDR (Time Domain Reflectometry). Bulletin of Engineering Geology and the Environment. vol. $59 \mathrm{n}^{\mathrm{C}}$ 3, 2000, p, 247-255.

Garcia Delgado R.A. - Influence of soil carbonates in lead fixation. I. Environ. SC Health, A31 (9), 1996, p. 2099-2109.
Gaudet J.-P. - Transfert d'eau et soluté dans les sols non saturés. Mesures et simulation. Thèse de doctorat es-sciences physiques, université de Grenoble, 1978.

Green W.H. Ampt G.A. - Studies on solls physics: I. The flow of air and water through soils, J. Agric. Sci., vol. 4, 1911. p. 1-24.

Jacobsen O.H., Schjonning P. - Field evaluation of time domain reflectometry for soll water measurements. $J$. of Hydrology , 151, 1993, p. 159-172.

Kachanoski R.G. Pringle E., Ward A.I. Field measurement of solute travel times using time domain reflectrometry. Soil Sci. Soc. Am. J., vol, 56, 1992, p. $47-52$.

Laryea K.B., Robin D.E. - Hydrodynamic dispersion insolving cationic absorption during unsaturated transient water flow in soil. Soil Sci. Soc. Am. J., vol. 46, 1982, p. $667-671$.

Meddahi M.E. Mallants D. Feven J. Baciii M., Vereecken H. - Paramètres essentiels d'écoulement des solutés dans un milleux poreux saturé: méthode de mesure et modèle d'interprétation. Sclence du sol, vol. 31, n² 4, 1993, p. 233-250

Mermoud A. - Propagation des substances miscibles dans un milieu poreux. Etude théorique et expérimentale. Bulletin technique de la Suisse romande, vol, 23, 1978, p. 341-345.

Mermoud A. - Contribution à l'étude des transferts simultanés d'eaut et de soluté en milieux poreux. Thèse $n^{\circ} 432$. EPFL. Suisse, 1982
Nadler A., Dasberg S., Lapid I. - Time Domain Reflectometry Measurement of Water and Electrical Conductivity of Layered Soil Column. Soil Sci. Soc Am. J. $n^{=55,1991, ~ p, ~ 938-943 . ~}$

Philip J.R. - The theory of infiltration. Advances in hydro sciences, vol. 5, 1969 . p. $215-296$

Richards L.A. - Capillary conduction of liquids through porous medium. Physics 1. 1931, p. 169-173.

Roth K., Schulin R. Flühler H., Attinger W. - Calibration of time domain reflectometry for water content measurement using a composite dielectric approach. Water Resour. Res., 16 (6), 1990, p. 961 979.

Rowe R., Chris J., Frank B. - Laboratory determination of diffusion and distribution coefficients of contaminants using undisturbed clayey soil. Can. Geotech. J.. n²5, 1988, p. 108-118.

Topp G.C. Davis J.L. Amman A.P. - Elec. tromagnetic determination of soil water content measurement in coaxial transmission lines. Water Ressour. Res., 16, 1980, p. $574-582$.

Van Genuchten M.-T. - A closed-form equation for predicting the hydraulic condictivity of unsaturated soils, Soil Sci. Soc. Am. I., vol. 44, 1980, p. 277-287.

Weiller K.W.. Steerihuis T.S., Boll J., Kung K.J.S. - Comparaison of ground penetrating radar and time domaine reflectometry as soil water sensors. Soil Sci. Soc. Am. J., n" 62, 1998, p. 1237-1239. 